\title{
FIRST MOLAR EXTRACTION IN CLASS III MALOCCLUSION IN ADULT PATIENTS
}

\section{Pricillia Friska Sianita.}

1. Lecturer, Orthodontic Department, Faculty of Dentistry Prof. Dr Moestopo University, Jakarta, Indonesia.

\section{Corresponding Author:}

Pricillia Friska Sianita

Lecturer, Orthodontic Department,

Faculty of Dentistry Prof. Dr Moestopo University

Jakarta, Indonesia

E-mail ID : ppsianita@gmail.com

Submitted Date :10-01-2020

Accepted Date :16-01-2020

\begin{abstract}
Background: Skeletal and dental Class III malocclusions have problems that are quite difficult for orthodontists who handle them and also for patients, especially female patients who experience them. This is mainly felt because the growth of the mandible continues even though the growth of the maxilla is over, so that as the patient ages, the facial features even get worse. Patient profile will appear concave and in terms of aesthetics will greatly disrupt the patient's self-confidence
\end{abstract}

Objective: A management of skeletal and dental Ill class malocclusion cases in adult patients will be discussed on this occasion mainly for the purpose of sharing experiences.

Material: Materials in this article is based on the case of class III malocclusions treated in a private clinic. Further study is supported by scientific information about similar cases from various journals and related textbooks.

Result: A satisfactory result of the patient can be obtained at the end of the treatment, especially in terms of its facial aesthetics.

Conclusion: In conclusion, it can be said that in certain cases, non-surgical orthodontic treatment is still possible to be performed in patients with skeletal and dental class II malocclusions with satisfactory facial correction.

Keywords: Class III malocclusion, skeletal, dental and facial pattern, extraction of first molars 


\section{INTRODUCTION}

A class III skeletal malocclusion must be divided into three categories based on maxillary abnormalities so that the case management can be arranged according to the problem at hand. The three categories in question are as follows: category $\mathrm{A}$ is the true mandibular prognatism which is understood as a condition with normal maxilla and the mandible shows excessive growth. Category B is a condition that shows unique characteristics with the maxilla and mandible both experiencing excessive growth accompanied by anterior cross bites. Category $\mathrm{C}$ is a condition characterized by hypoplastic maxilla accompanied by anterior cross bites. Management in each category should be focused on the problems faced.

If a Class III skeletal malocclusion is assessed from the maxillary position, then Class III dental malocclusion, which generally accompanies this Class Ill skeletal relation, is assessed based on the relation of the first molar according to the Angle classification. Angle class III malocclusion is understood as an occlusion condition characterized by the mandibular first molar being positioned more anteriorly in relation to the maxillary first molar (figure 1). Angle classification is still the most widely used classification for malocclusion because of its simplicity. However, quite a lot of criticism raised by experts on the classification which only evaluates relations in the antero-posterior or sagittal direction because it does not consider relations in the transversal or vertical direction. Angle classification also does not discuss the relationship of the teeth with facial soft tissue (profile). The molar relationship in the antero-posterior direction is very important because in general, the management of class III malocclusion is sought to achieve the relationship of class I molar teeth with normal overbite and overjet. In this case, the relationship of Class I molar teeth is characterized by the mesio-buccal cusp position of the maxillary permanent first molar teeth in occlusion with the buccal groove of the mandibular permanent first molar. In the case of Class Ill malocclusions experienced by adult patients, the achievement of class I molar relationships such as this can be determined as a treatment goal regardless of the maxillary and mandibular position or skeletal pattern, especially in adult patients with class III skeletal malocclusion who refuse orthognathic surgical management to obtain good skeletal relationships.
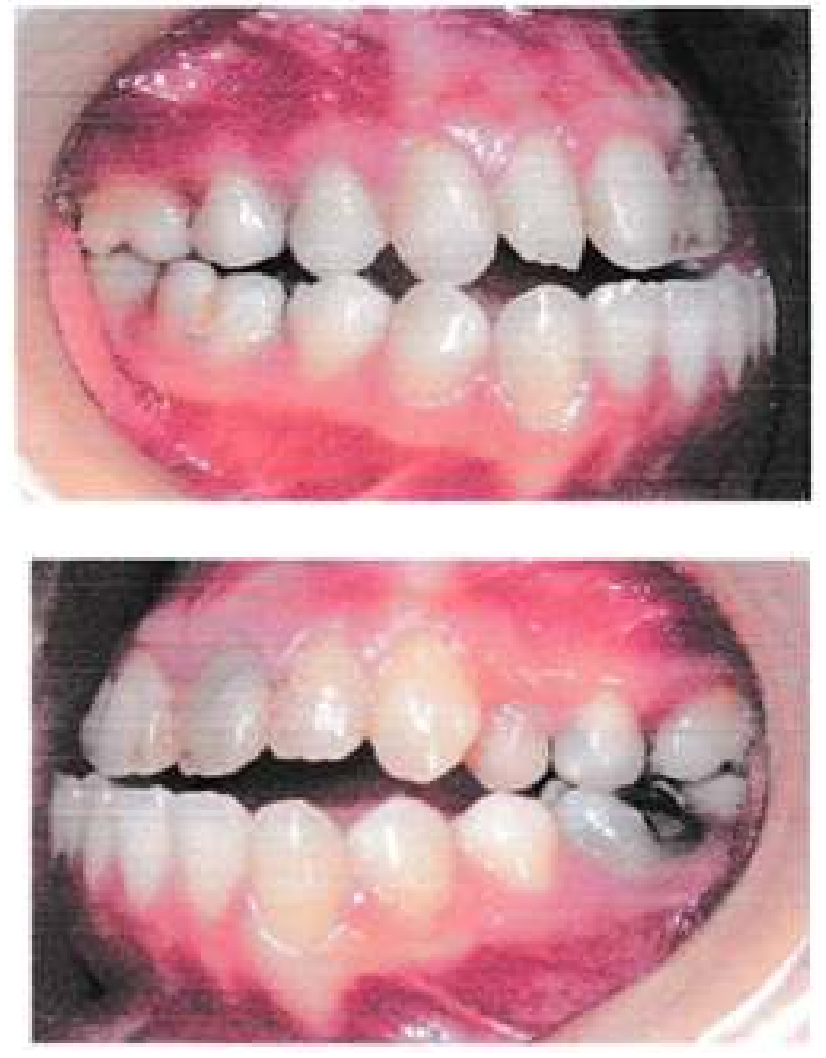

Fig 1. The pattern of molar relationships in Class Ill malocclusion, it appears that the mandibular first molars are more anterior than the maxillary first molars. (Documentation of private collections)

In addition to the maxillary position and the relation of the first permanent molar, class III malocclusion can also be assessed from its facial pattern. In this case, AM.Schwarz divides normal face profit into three categories. ${ }^{1-3}$ The first category is a facial pattern called ante-face, which is facial profile characterized by the position of the lower anterior face which is relatively protrusive towards the perpendicular line of the soft tissue nasion point (figure 2a-c). The second category is the facial pattern called the mid-face with the characteristic position of the lower anterior face being approximately at the vertical/ perpendicular line of the soft tissue nasion point. The third category is the facial pattern called retro-face with the characteristic position of the lower anterior face behind the perpendicular line of the soft tissue nasion point.

According to Park, JU et al, mid-face facial patterns are normal characteristics for Asians while ante-face facial patterns are normal characteristics in Africans. However, at this time, that view has changed. Both men and women want retro-face facial patterns and tend to choose three-dimensional face patterns over flat face patterns. 


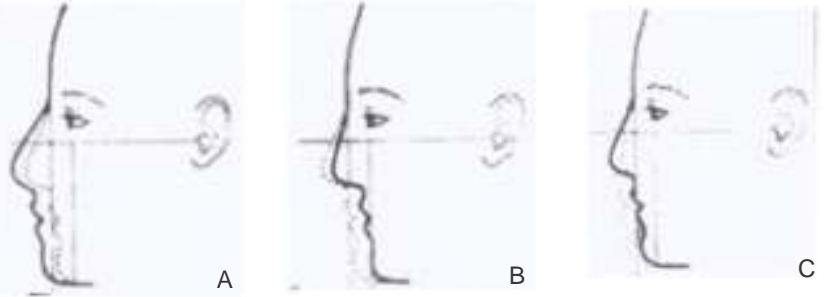

Fig 2a-c. The division of facial pattern categories according to A.M.Schwarz, namely: $a$ is an ante-face facial pattern, $b$ is a retro-face facial pattern and $c$ is a mid-face facial pattern

The three-dimensional face pattern that is meant here is a face that has three concavities (figure 3 ).

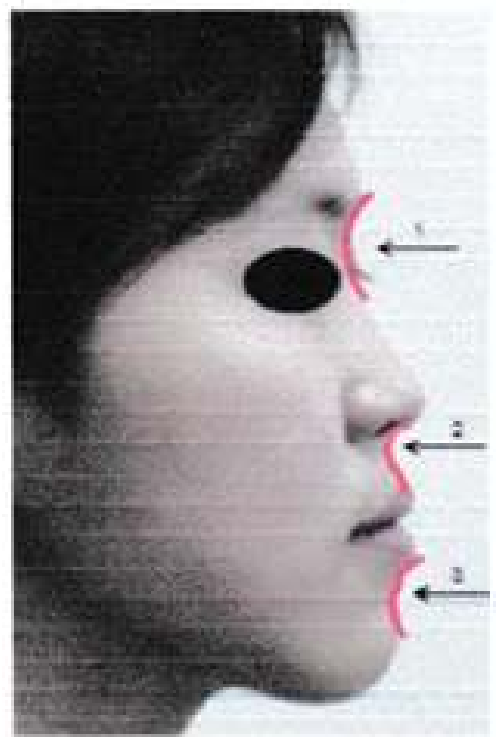

Fig 3. Three-dimensional facial patterns with three concavities in three areas are more desirable by patients today. The first is called frontonasal concavity, the second is called nasolabial concavity and the third is called labiomental concavity. (personal collection documentation)

The various classifications of malocclusion which include skeletal and facial patterns will serve as a guide for dentists to focus on the treatment goals to be achieved as well as the procedures involved including orthognathic surgical procedures to achieve better results. Beyond the ideal treatment goals, there is a treatment that we call compromise. This is especially true in skeletal malocclusions that occur in the post-growth period age and treated without surgery. In this case, the patient's dental occlusion is strived to achieve the final result without changing the skeletal pattern. Of course this approach must be accompanied by careful consideration of several factors related to balance or stability to prevent relapse.

\section{TOOTHEXTRACTION}

Cases of malocclusion that have severe crowding characteristics in some cases will require the extraction to eliminate the crowding condition. Extraction is not the only way to get a room in an effort to eliminate crowding. Some other ways include expansion, distalization and also interproximal reduction (IPR). Tooth extraction is generally chosen, especially in cases with large space requirements. Related to this extraction, the teeth that are generally chosen are the first or second premolars. In class III malocclusions, the most commonly extraction is the right and left first premolars in the lower jaw and the right and left second premolars in the upper jaw. However, in certain considerations other teeth such as the lower jaw incisor or the first permanent molar may have been selected. Consideration of severe dental caries is believed to be better than the extraction of healthy teeth without caries. In cases of extraction such as this, it is necessary to conduct a comprehensive examination of the status of other teeth to ensure that the second molar shows a good developmental tendency and there are no eruptive abnormalities or other missing teeth..$^{7-8}$

The purpose of writing this article is to share the experience in the management of Class Ill malocclusions that have been treated with the extraction of right and left mandibular first permanent molar and fixed appliance full arch.

\section{CLINICAL CASE}

$\mathrm{LN}$ is a 21-year-old chineesefemale patient, college student, comes with the main complaint of a face profile and smile which makes her uncomfortable. Extra oral, the patient shows antefacial face pattern (figure 4) with a hyperdivergent growth pattern (figure 5). The patient's intra oral shows class III malocclusion both for molar and canine relations (figure 1). The patient has a reverse overjet approaching $12 \mathrm{~mm}$ with an anterior openbite. Analysis of the lateral cephalometry shows a class III skeletal classification called true prognathism because of SNA 800 and SNB 840. So the patient's maxilla is normal but the mandible shows excessive growth, and the patient's ANB is -4 - (figure 5). The axial inclination of the upper incisor is proclination with an IRA-NA angle of $41^{\circ}$ as well as the axial inclination of the lowerincisors at $33^{\circ}$. Overall, patients have problems in three spatial dimensions, 
namely sagittal or antero-posterior which are characterized by class III relations in molar and canine teeth, vertically marked by open bite and transversal which are marked by anterior and posterior cross bites.

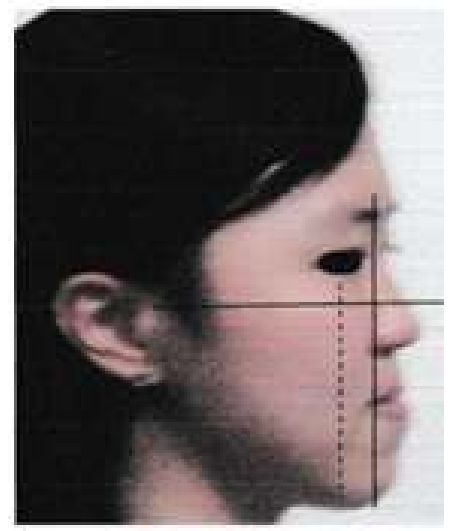

Fig 4. An ante-face pattern with characteristics of a relatively protrusive lower anterior face position or in

front of a line perpendicular to the soft tissue nasionpoint. (personal collection documentation).

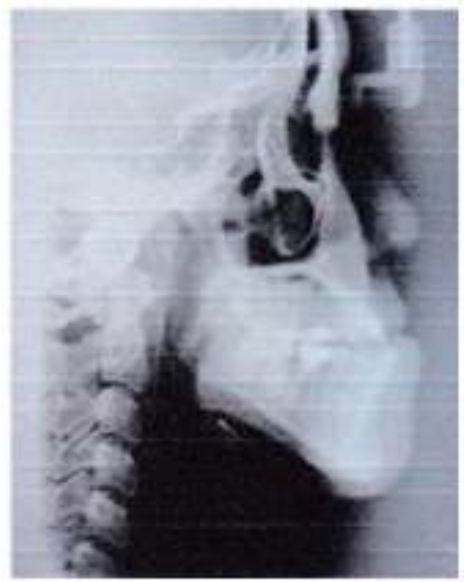

Fig 5. The patient's lateral cephalogram shows a hyperdivergen growth pattern with a Frankfort

Mandibular angle of $35^{\circ}$ (high angle).

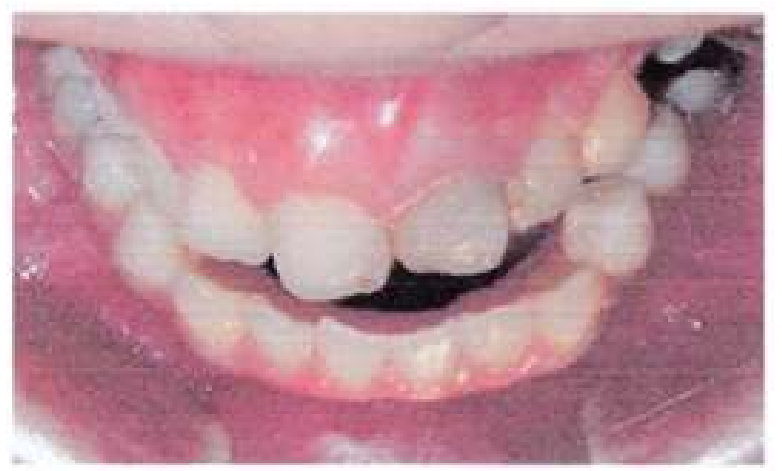

Fig 6. The patient has anterior and posterior cross bites (private collection documentation).
Panoramic roentgenogram of the patient showed the condition of the first molars that had been severely damaged with large restoration (garnbar 7). Both the second permanent molar and the third permanent molar have erupted in better condition than the first permanent molar.

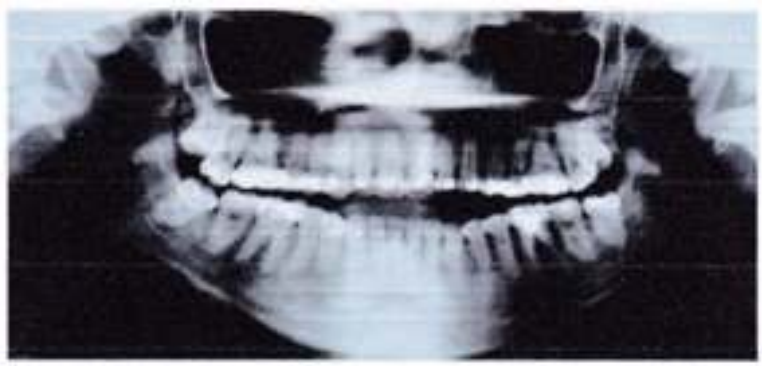

Fig 7. The patient panoramic shows the condition of posterior teeth which is not good - has a large restoration.

Based on the results of cephalometric analysis and study models, patient diagnosed with class III skeletal malocclusion due to mandibular prognathism and hyperdivergent growth patterns, class III dental angles with ante-face facial patterns. Patient treatment plans are established as a form of compromise approach because of his refusal of orthognathic surgical approach. For this reason, treatment goals are designed for fixed braces full arch with extraction of right and left first permanent molars for overjet and overbite correction (figure 8). The removal of the first permanent molar in this case was done with the consideration that there had been a large restoration, one of the molar teeth (tooth 36) had undergone root canal treatment and radiographically had shown periodontal involvement while the second and third molar teeth had erupted. ${ }^{8}$ Posterior cross bite will be overcome through archwire expansion. The use of class III elastic is also added in order to achieve satisfactory treatment results. Patient wears Roth prescription brackets with .018 slot. Archwire sequences in this case begin with .012 superelasticNiTi wire for initial alignment and leveling of the upper and lower arches, followed by Australian wire .018 to do archwire expansion and cross bite correction. Space closing mechanics in this case are done in stages including the application of moderate anchorage, use of power chains and class III elastic with rectangular SS 16x22 archwire for overjet and overbite correction. 

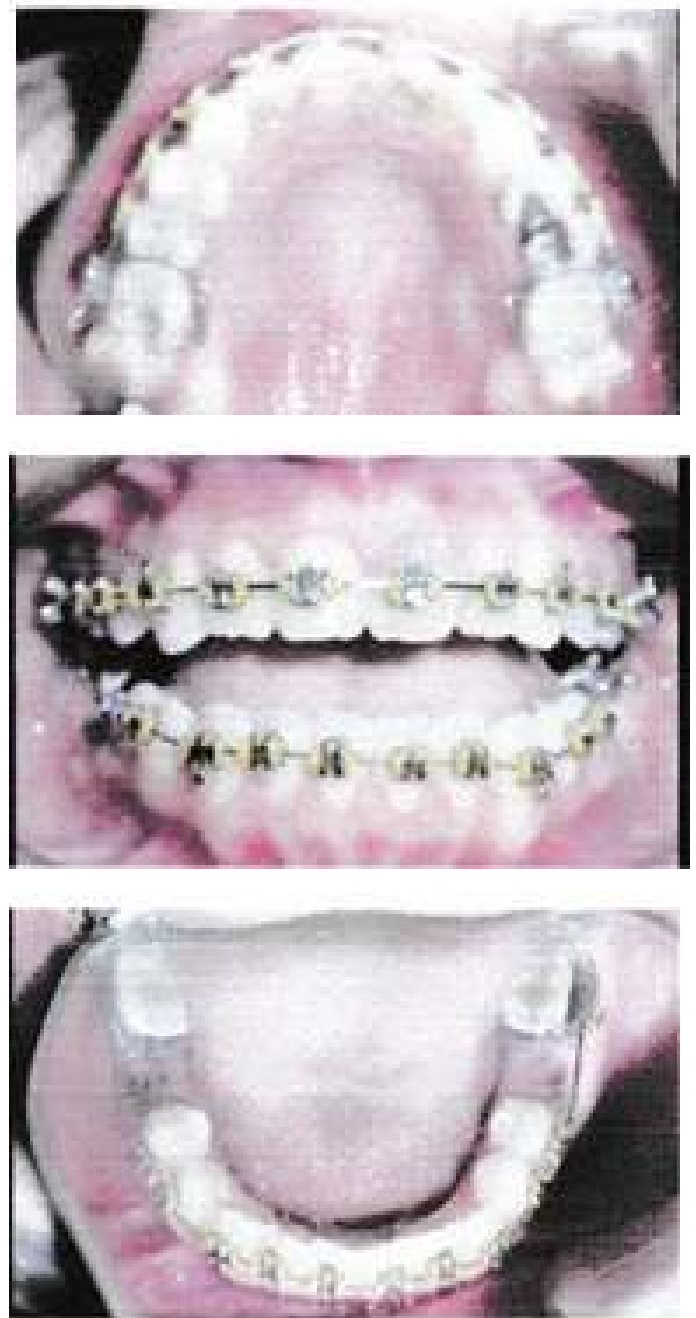

Fig 8. Treatment performed with fixed braces full arch accompanied by extraction of the mandibular first permanent molar.

Post-treatment can be seen in Figure 9, the patient has an occlusion with a class I molar and canine relationship. Overjet and overbite are also corrected. The second permanent molar is allowed to move mesially to close part of the space produced by the extraction of the mandibular first permanent molar. This treatment produced a noticeable change in the facial profile (figure 10a). Post-treatment lateral cephalogram analysis showed unchanged SNA and SNB values, as well as ANB values. The IRA-NA angle also did not change but this was not the case with the IRB-NB angle which has changed to $24^{\circ}$ from before treatment at $33^{\circ}$. This means that overjet correction is mainly achieved by retraction of the anterior segment posteriorly and the former maxillary permanent molar tooth extraction space is utilized partly by the movement of the posterior segment to anterior and partly by the anterior segment to the posterior(moderate anchorage).

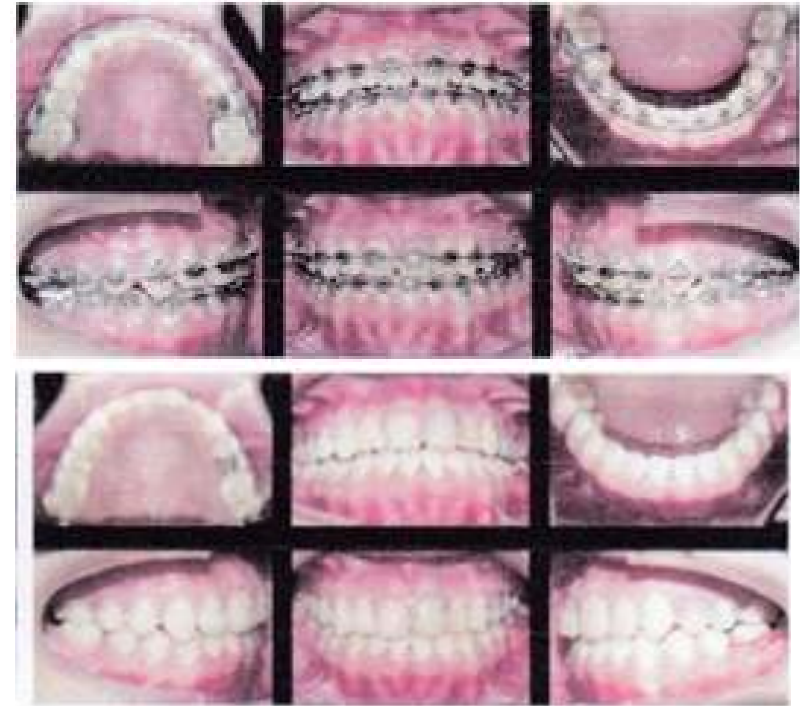

Fig 9. The patient's occlusion condition just before bracket removal (left) and the patient's occlusion condition after bracket removal (right). (private collection documentation). Also seen here that the space is closed properly, the overjet and overbite have also been corrected as well.
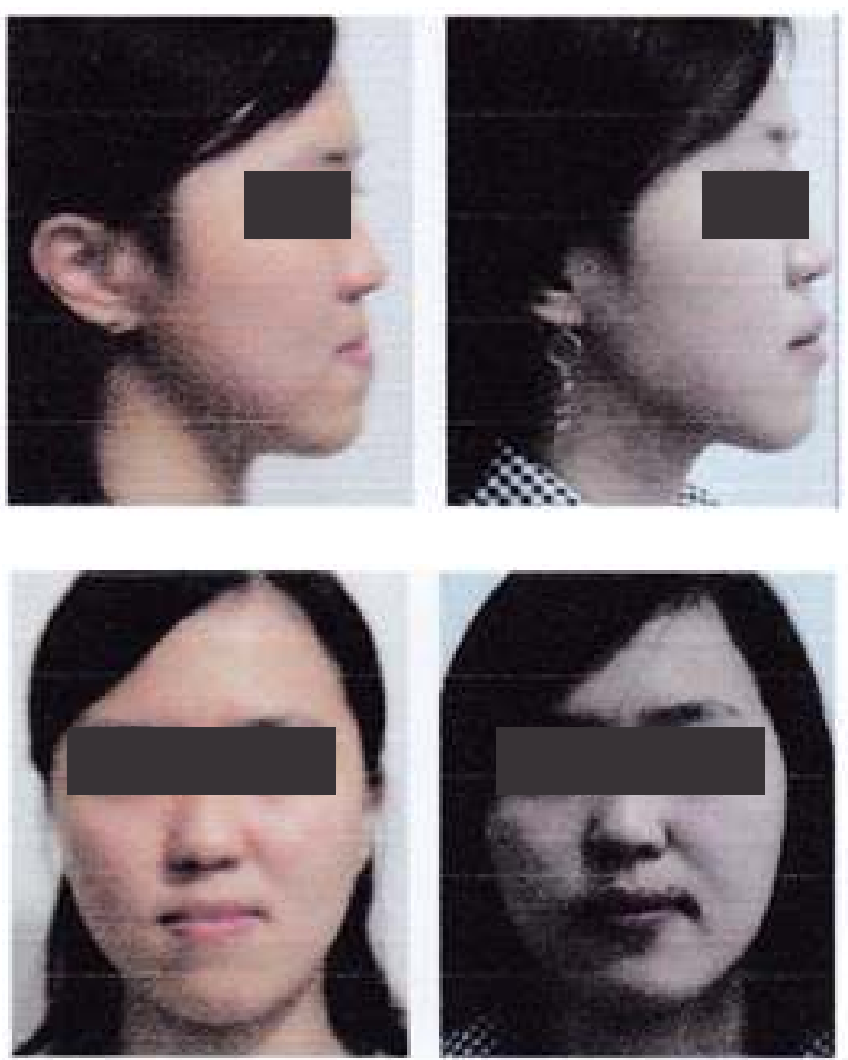

Fig 10.a) The patient's profit appears to change (left) before treatment and (right) after treatment. b) frontal view of the patient's face also shows changes (left) before treatment and (right) after treatment.. 


\section{DISCUSSION}

Cases of class III malocclusion that occur in adult patients who have passed the period of facial growth will face limited treatment options. Generally, orthognatic surgery besides orthodontic treatment will be the main consideration if the patient wants an ideal result. However, if the patient refuses surgery, compromise orthodontic treatment will require the extraction of teeth. Until now, nonsurgical orthodontic treatment in class III malocclusion which is believed to be the best option is fixed appliance with tooth extraction. So the correction is limited to dentoalveolar compensation without correction for the skeletal pattern. ${ }^{10,11}$

Management of Class III malocclusion cases with the choice of extraction like this is faced with several considerations, which are complicated orthodontic mechanics, long treatment time and the opportunity to achieve the relationship of class I molar and canine even for the lower jaw, where second permanent molars that will replace the position of the first permanent molars to achieve class I relationship with maxillary first permanent molar. This is possible with the help of a moderate anchoring model. Moderate anchorage by power chain allows the posterior segment to move mesially while retraction of the anterior segment posteriorly is performed to correct the patient's overjet. Anterior segment retraction and overjet correction ultimately help improve the lower anterior face, resulting in a labiomentalconcavity. Another orthodontic mechanic involved in closing the space of the former permanent molar tooth extraction, which also helps retract the anterior segment posteriorly is the use of class III elastic. The action of using this class III elastic, in addition to helping retraction of the anterior lower segment to move posteriorly, also helps move the upper lip forward / anterior to accentuate the nasolabial concavity. This improvement in nasolabial and labiomental concavity will support the improvement of the patient's three-dimensional facial profile. In the management of non-surgical class III skeletal and dental malocclusions in adult patients, improvement of the facial profile is very important and help determines the level of patient satisfaction. In other words, the achievement of patient satisfaction can also be interpreted as a successful treatment for orthodontists.

\section{CONCLUSION}

The management of class III malocclusions that have a skeletal, dental and soft tissue component in adult patients generally involves orthognathic surgical procedures to obtain aesthetically good results. However, not all patients are prepared for orthognathic surgical procedures even though they promise better aesthetic quality in the final outcome. In such cases, compromise treatment in the form of dentoalveolar compensation and fixed appliance full arch remains an option that should be considered. This case report has proven that non-surgical treatment with extraction of mandibular first permanent molars is capable of producing an improvement in the patient's three-dimensional face profile and occlusion with class I molar and canine. Relationship.

\section{ACKNOWLEDGMENTS}

The author expresses his highest appreciation and sincere thanks to the patient and all related parties who have helped the publication of this article.

\section{Conflict Of Interest}

There is no conflicts of interest regarding the publication of this article.

\section{REFERENCES}

1. Park,JU.,Baik,SH., Classification of Angle Class III malocclusion and its treatment modalities. Int.J.Adult.Orthod.Orthognati Surg. 2001; 16:19- 29.

2. Van Lcon,JAVV., A New . Method for Indicating normal and abnormal relationship of the teeth to the facial lines. Dent.Cosmos.1915;57:973-983,10931101,1229-1235.

3. Obwegeser,H., Profile Planning Based on Alteration in the Position of the Bases of the Facial thirds. J.OralMaxillofac.Surg. 1986;44:302- 311.

4. Seddon,JL., Extraction of four first molars: a case for a general practitioner?.Journal of Orthodontics, 2004. Vol.31,no 2, pp 80-85. 
5. Lin,J., Gu,Y., Lower second molar extraction in correction of severe skeletal class III malocclusion. The Angle Orthodontist. 2006. Vol. 76, no 2, pp.217-225.

6. Saito,I.,Yamaki,M.,Hanada,K., Nonsurgical treatment of adult open b i t e u sing edgewise appliance combined with high-pull headgear and class III elastics. The Angle Orthodontists. 2005. Vol.75, no 2, pp.277283.

7. Bennet,JC., McLaughlin,RP., Orthodontic Management of the Dentition with Preadjusted Appliance. Toronto, Mosby. 2003, pp. 292

8. Schroeder, MA., Schroeder, DK., Santos, DJS., Leser, MM., Molar e x t r a c t i o n s i n orthodontics. Dental Press Journal of orthodontics, 2012, Vol. 16, no 6, pp. 130152.

9. Gili,D.,Naini,F.,McNaily,M.,Jones,A., The Management of Transverse $\mathrm{M}$ a x i 11 a r $\mathrm{y}$ Deficiency. Dent Update 2004;31:516-523.

10. Ruellas,AC., Baratieri,C., Roma,MB., et.al..Angle class III malocclusion tre ate d with mandibular first molar extractions. 2012. Am. J. Orthod Dentofac. Orthop. Vol. 142, no 3, pp. 384-392.

11. Janson,G., Prado De Souza, JE., De Andrade Alves, F., et al. Extreme dentoalveolar compensation in the treatment of class III malocclusion. 2005. A m. J . O r t h o d . Dentofac. Orthop. Vol. 128, no 6, pp. 787-794 